\title{
Brain-Derived Neurotrophic Factor Mediates the Activity- Dependent Regulation of Inhibition in Neocortical Cultures
}

\author{
Lana C. Rutherford, Andrew DeWan, Holly M. Lauer, and Gina G. Turrigiano \\ Department of Biology and Volen Center for Complex Systems, Brandeis University, Waltham, Massachusetts 02254
}

The excitability of cortical circuits is modulated by interneurons that release the inhibitory neurotransmitter GABA. In primate and rodent visual cortex, activity deprivation leads to a decrease in the expression of GABA. This suggests that activity is able to adjust the strength of cortical inhibition, but this has not been demonstrated directly. In addition, the nature of the signal linking activity to GABA expression has not been determined. Activity is known to regulate the expression of the neurotrophin brain-derived neurotrophic factor (BDNF), and BDNF has been shown to influence the phenotype of GABAergic interneurons. We use a culture system from postnatal rat visual cortex to test the hypothesis that activity is regulating the strength of cortical inhibition through the regulation of BDNF. Cultures were double-labeled against GABA and the neuronal marker MAP2, and the percentage of neurons that were GABA-positive was determined. Blocking spontaneous activity in these cultures reversibly decreased the number of GABA-positive neurons without affecting neuronal survival. Voltage-clamp analysis of inhibitory currents demonstrated that activity blockade also decreased GABA-mediated inhibition onto pyramidal neurons and raised pyramidal neuron firing rates. All of these effects were prevented by incubation with BDNF during activity blockade, but not by neurotrophin 3 or nerve growth factor. Additionally, blockade of neurotrophin signaling mimicked the effects of activity blockade on GABA expression. These data suggest that activity regulates cortical inhibition through a BDNF-dependent mechanism and that this neurotrophin plays an important role in the control of cortical excitability.

Key words: BDNF; visual cortex; dissociated culture; activitydependent; inhibition; GABA; interneurons
Maintaining the correct balance of excitation and inhibition is crucial for the proper functioning of cortical circuits (Kriegstein et al., 1987; Chagnac-Amitai et al., 1989), suggesting that this balance should be tightly regulated both during development and in adulthood. The excitability of cortical circuits is modulated by interneurons that release the inhibitory neurotransmitter GABA. In primate and rodent visual cortex, expression of GABA is influenced by visual input; dark-rearing or TTX injection into one eye decreases the number of GABA-immunopositive neurons in primary visual cortex (Hendry and Jones, 1986; Benevento et al., 1995). This suggests that activity is able to adjust the strength of cortical inhibition, but this has not been demonstrated directly.

What is the signal linking activity to the regulation of cortical GABA expression? An interesting candidate for this signal is the neurotrophin brain-derived neurotrophic factor (BDNF). The neurotrophins are a class of factors, including BDNF, nerve growth factor (NGF), and neurotrophin 3 (NT3), that support the survival and differentiation of a variety of peripheral and central neurons (for review, see Lindsay et al., 1994). BDNF expression in postnatal cortex is tightly regulated by neuronal activity. Seizure induction in vivo or depolarization by high potassium in culture increase cortical BDNF expression (Isackson et al., 1991; Ghosh et al., 1994). There is a sharp increase in BDNF expression in visual cortex after eye opening (Maisonpierre et al., 1990), and

Received Jan. 17, 1997; revised March 26, 1997; accepted March 28, 1997.

This work was supported by National Science Foundation Grant IBN-9421233, the Whitehall Foundation, National Institutes of Health Grant K02 NS-01893, and the Sloan Center for Theoretical Neurobiology. We thank Amgen, Incorporated, (Thousand Oaks, CA) for providing us with NT3. We thank Susan Birren and Sacha Nelson for useful discussions and for their critical reading of this manuscript.

Correspondence should be addressed to Dr. Gina G. Turrigiano, Department of Biology, Brandeis University, Waltham, MA 02254.

Copyright (C) 1997 Society for Neuroscience $0270-6474 / 97 / 174527-09 \$ 05.00 / 0$ dark-rearing decreases BDNF expression in rat visual cortex both during development and in adulthood (Castrén et al., 1992). A role for BDNF has now been suggested in a number of cortical processes (Ghosh et al., 1994; Cabelli et al., 1995; McAllister et al., 1995, 1996). BDNF can increase the GABA content and level of GAD activity in GABAergic neostriatal neurons in culture (Mizuno et al., 1994; Ventimiglia et al., 1995), influence peptide expression in hippocampal interneurons (Marty et al., 1996a,b), and increase GABA uptake and soma size in embryonic cortical interneurons (Widmer and Hefti, 1994). These studies suggest that BDNF can influence transmitter expression in several populations of central interneurons.

These observations raise the possibility that activity regulates inhibition in visual cortical circuits through the regulation of BDNF. We use a postnatal culture system from rat visual cortex, containing both excitatory pyramidal neurons and inhibitory interneurons, to test this hypothesis. We show that blockade of spontaneous activity reversibly decreases the number of neurons that are immunopositive for GABA. This decrease in GABA expression is correlated with a decrease in inhibition between interneurons and pyramidal neurons and an increase in pyramidal neuron firing rates. These effects of activity blockade can be prevented by BDNF, but not by NGF or NT3, and blockade of neurotrophin receptor signaling with $\mathrm{K} 252 \mathrm{a}$ mimics the effects of activity blockade. These data suggest that activity continuously adjusts cortical inhibition and pyramidal neuron firing rates by regulating the production of BDNF.

\section{MATERIALS AND METHODS}

\section{Cell cultures}

Cultures from postnatal rat visual cortex were prepared using a modification of the technique of Huettner and Baughman (1986, 1988). Rat pups between postnatal days 4 and 6 were anesthetized using isofluorane 
and decapitated. The brain was removed, and a portion of occipital cortex corresponding to visual cortex was removed and placed into sterile artificial cerebral spinal fluid (ACSF) containing (in $\mathrm{mM}$ ): $126 \mathrm{NaCl}, 3$ $\mathrm{KCl}, 2 \mathrm{CaCl}_{2}, 2 \mathrm{MgSO}_{4}, 1 \mathrm{NaHPO}_{4}$, and $25 \mathrm{NaHCO}_{3}$, equilibrated with $5 \% \mathrm{CO}_{2} / 95 \% \mathrm{O}_{2}$ ). The tissue was minced and the ACSF was withdrawn, and enzyme solution composed of $25 \mathrm{U} / \mathrm{ml}$ papain, $1 \mathrm{~mm}$ L-cystein, and $0.5 \mathrm{~mm}$ EDTA (Worthington Biochemical, Freehold, NJ) in Earle's balanced salt solution (EBSS, Life Technologies, Gaithersburg, MD) was added and the tissue was incubated for $1.5 \mathrm{hr}$ in a $5 \% \mathrm{CO}_{2}$ incubator at $37^{\circ} \mathrm{C}$. The tissue was rinsed in a weak trypsin inhibitor solution $(1 \mathrm{mg} / \mathrm{ml}$ ovomucoid and $1 \mathrm{mg} / \mathrm{ml} \mathrm{BSA}$ in sterile EBSS) and gently triturated, and a strong inhibitor solution $(10 \mathrm{mg} / \mathrm{ml}$ ovomucoid and $10 \mathrm{mg} / \mathrm{ml} \mathrm{BSA}$ in sterile EBSS) was added and the suspension centrifuged at low speed for $5 \mathrm{~min}$. The supernatant was withdrawn, and the pellet was resuspended in $5 \mathrm{ml}$ of growth medium (see below) and plated at a density of $\sim 100,000$ cells $/ \mathrm{cm}^{2}$ onto glass-bottom $35 \mathrm{~mm}$ culture dishes previously coated with rat tail collagen. Growth medium consisted of MEM (Life Technologies) with $5 \%$ fetal bovine serum, $1 \%$ N2 supplement (Life Technologies), 30 $\mathrm{mm}$ dextrose, $200 \mathrm{~mm}$ L-glutamine, and $50 \mathrm{U} / \mathrm{ml}$ penicillin/streptomycin, with a final osmolarity between 310 and 320 mOsM. Cultures were incubated in a $5 \% \mathrm{CO}_{2}$ incubator at $37^{\circ} \mathrm{C}$ until use. Cultures were fed every 3-4 d by replacing $1 \mathrm{ml}$ of medium with fresh medium. After 3-5 d in vitro, when non-neuronal cells became confluent, cell proliferation was inhibited by adding $10 \mu \mathrm{M}$ 1-gamma-D-arabinofuranosyl cytosine.

\section{Electrophysiology}

Current-clamp recordings. For electrophysiological recordings, cultures were moved to the stage of an inverted Nikon Diphot microscope and superfused continuously with ACSF $+20 \mathrm{~mm}$ dextrose at room temperature, with an osmolarity of 305-310 mOsM. The ACSF was bubbled continuously with $5 \% \mathrm{CO}_{2} / 95 \% \mathrm{O}_{2}$. The preparation was grounded with a silver/silver chloride ground wire connected to the headstage of an Axoclamp 2B. Whole-cell recordings were obtained using patch pipettes (3-5 $\mathrm{M} \Omega$ resistances) filled with intracellular solution consisting of (in mM): $130 \mathrm{KMeSO}_{4}, 10 \mathrm{KCl}, 10 \mathrm{~K}$-HEPES, $2 \mathrm{MgSO}_{4}, 0.5 \mathrm{EGTA}$, and 3 ATP; final osmolarity was adjusted to $290-295 \mathrm{mOsM}$ with sucrose. Seal resistances were 3-6 G $\Omega$, and series resistances were 5-15 M $\Omega$ and were left uncompensated. The amplifier was controlled, and data were acquired using the Pulse Control software (J. Herrington, K. Newton, and $\mathrm{R}$. Bookman, University of Miami) running on a PowerMac 7100 interfaced with the amplifier using an ITC 16 board (Instrutech, Great Neck, NY). Neurons with resting potentials of less than $-50 \mathrm{mV}$, input resistances of $<700 \mathrm{M} \Omega$, or recordings with series resistances $>20 \mathrm{M} \Omega$ were discarded. For recording purposes, bipolar interneurons were identified morphologically, based on a distinctive oval shape to the soma with two prominent dendrites emerging from the long axes (see Fig. 2D).

Voltage-clamp recordings. Synaptic currents were recorded as described above, except that the amplifier used was an Axopatch $1 \mathrm{~B}$ in voltageclamp mode, and the internal solution contained $130 \mathrm{mM} \mathrm{CeMeSO}_{4}$ in place of $\mathrm{KMeSO}_{4}$ to block potassium currents, $10 \mathrm{~mm}$ EGTA, and $10 \mathrm{~mm}$ QX-314 to block generation of action potentials. This allowed neurons to be voltage-clamped to $0 \mathrm{mV}$ (the reversal potential of the excitatory currents) so that large, outward GABA-mediated currents could be recorded in isolation. Each neuron was voltage-clamped to $-70 \mathrm{mV}$ and then stepped up to $0 \mathrm{mV}$ for $30 \mathrm{sec}$ recording periods interspersed with $30 \mathrm{sec}$ rest periods at $-70 \mathrm{mV}$. Four minutes of data was obtained and averaged for each neuron. For each recording, series resistance and input resistance were continuously monitored, and if these values changes by $>15 \%$, the data were discarded, as were recordings with series resistances of $>20 \mathrm{M} \Omega$.

\section{Immunohistochemistry}

Cultures were processed for indirect immunofluorescence against GABA and the neuronal marker MAP2. Cultures were fixed for $10 \mathrm{~min}$ using a $4 \%$ paraformaldehyde, $5 \%$ sucrose solution in $0.2 \mathrm{M}$ phosphate buffer. Cultures were washed three times in PBS $(137 \mathrm{~mm} \mathrm{NaCl}, 3 \mathrm{~mm} \mathrm{KCl}$, in 0.2 $\mathrm{M}$ phosphate buffer). They were then blocked and permeabilized in $10 \%$ goat serum and $0.1 \%$ Triton X-100 in PBS for $20 \mathrm{~min}$. The cultures were then incubated overnight in primary antibody in $10 \%$ goat serum in PBS (PBS-G). Cultures were rinsed three times with PBS and washed for $1 \mathrm{hr}$ in PBS-G. The cultures were incubated in the secondary antibodies in PBS-G for $1 \mathrm{hr}$ at room temperature, then washed for $2 \mathrm{hr}$ in PBS, serially dehydrated in ethanol, and rinsed two times in xylene. They were mounted in DPX (Electron Microscopy Sciences, Fort Washington, PA) and coverslipped and allowed to dry before examination.
To double-label cultures against both GABA and MAP2, cultures were incubated concurrently in a rabbit anti-GABA polyclonal antiserum (Sigma, 1:800) and a mouse monoclonal anti-MAP2 antibody (Sigma, 1:500). Secondary antibodies were a rhodamine-conjugated goat anti-rabbit and a fluorescein-conjugated goat anti-mouse (Boehringer-Mannheim, Indianapolis, IN, both used at concentrations of 1:50). Preabsorption of the anti-GABA antiserum for $4 \mathrm{hr}$ with $10^{-4} \mathrm{M}$ GABA completely abolished staining, whereas staining was unaffected by preabsorption with $10^{-4} \mathrm{M}$ glutamate. No staining was observed when the primary antibodies were omitted from the protocol. Cultures were photographed using a laser scanning Bio-Rad MRC 600 Confocal microscope (Hercules, CA) equipped with rhodamine and fluorescein optics.

\section{Cell counts and physiological data analysis}

Cultures were examined and counted using a Nikon Diphot inverted microscope equipped with fluorescence optics. Cell counts were accomplished by counting the number of immunostained neurons in consecutive fields of view in two independent strips through the center of each dish. Results for the two strips were then averaged. Each field of view was counted first for the total number of MAP2-positive neurons using fluorescein filters and then for the total number of GABA-positive neurons using rhodamine filters. For each dish, the number of GABApositive neurons/strip was divided by the total number of neurons/strip to give the percent of GABA-positive neurons for each dish. Only dishes in which there were $>100$ neurons/strip were included, although results for less dense cultures were not significantly different. Among the dishes with $>100$ neurons/strip, neuronal densities did not vary significantly among conditions and averaged $62 \pm 12$ neurons $/ \mathrm{mm}^{2}$. All numbers are expressed as the mean \pm SEM for the number of cultures (for cell counts) or the number of neurons (for physiological recordings) indicated. Each manipulation was tested on cultures from at least two different platings. For each experiment, controls from sister cultures were run in parallel. To analyze spike frequency data, the number of spontaneous action potentials generated in a 4 min period was measured, and this number was then expressed as spikes/min. To accurately measure resting potentials and input resistances, these values were measured with synaptic transmission blocked with the AMPA receptor antagonist CNQX $(50 \mu \mathrm{M})$ and the $\mathrm{GABA}_{\mathrm{A}}$ receptor antagonist bicuculline $(10 \mu \mathrm{M})$. To analyze synaptic current data, the total area under the outward current transients was integrated for each $30 \mathrm{sec}$ sweep of data. This value was averaged over eight such $30 \mathrm{sec}$ sweeps to give a measure of the outward current per unit time for each neuron.

For wash experiments, cultures were treated with TTX for $2 \mathrm{~d}$, and the TTX was then washed out through seven exchanges of $1 \mathrm{ml}$ of medium with fresh control medium. Control cultures were washed identically to experimental cultures and fixed and processed in parallel. A stock solution of TTX (Sigma) was made in sterile $\mathrm{H}_{2} \mathrm{O}$ and kept at $4^{\circ} \mathrm{C} ; 2 \mu \mathrm{l}$ was added to each treated culture for a final concentration of $0.1 \mu \mathrm{M}$. TTX was refreshed after $24 \mathrm{hr}$. For some control cultures, vehicle was added at the same concentration and had no effect on GABA staining. Human recombinant BDNF, NGF, and NT3 were obtained from the following sources: BDNF, from Promega, Hercules, CA; NGF, from Upstate Biotechnology (Lake Placid, NY); and NT3, from Amgen (Thousand Oaks, CA). Aliquots of stock solutions of BDNF, NGF, and NT3 in $1 \mathrm{mg} / \mathrm{ml}$ $\mathrm{BSA}$ were kept at $-80^{\circ} \mathrm{C}$ until use, then thawed and added to the cultures at the indicated concentration. Neurotrophins were not refreshed on subsequent days. K252a was added at concentrations of 10 , 50, or $200 \mathrm{nM}$ and was refreshed after $24 \mathrm{hr}$.

\section{RESULTS}

\section{Activity blockade reduces the percentage of GABA- positive neurons in cortical cultures}

Cortical cultures were plated between postnatal days 4 and 6 and were maintained in culture for 1-2 weeks before experimentation. During this time, the neurons adhered to the substrate, extended processes, and formed synaptic connections. Whole-cell recordings showed that by $5 \mathrm{~d}$ in vitro, spontaneous synaptic potentials could be detected (Fig. $1 \mathrm{~A}$, arrow), which periodically brought the neurons over threshold for firing action potentials. This activity was completely blocked by the sodium channel antagonist TTX $(0.1 \mu \mathrm{M})$ (Fig. 1B). 


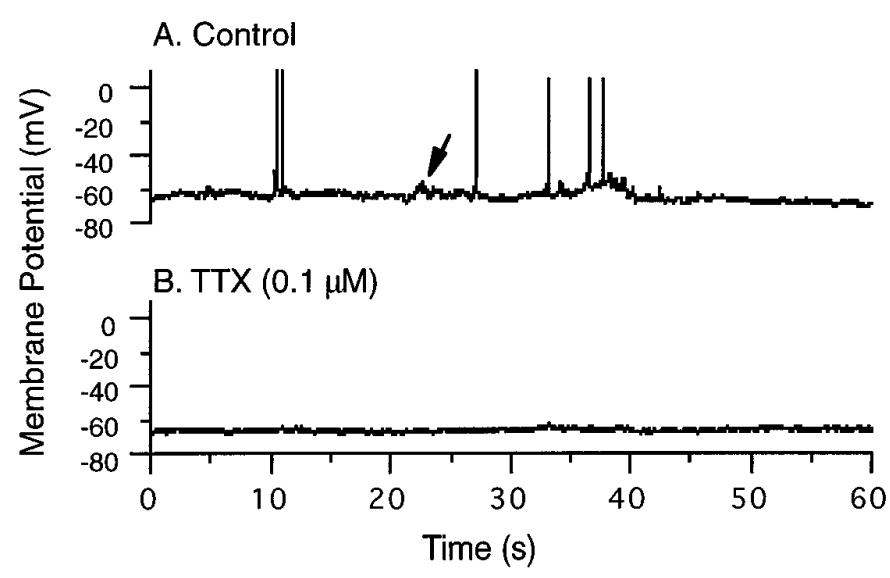

Figure 1. Spontaneous activity of cortical neurons in culture. A, Control, A whole-cell recording from a cortical pyramidal neuron after $5 \mathrm{~d}$ in vitro. Depolarizing synaptic potentials (arrow) could be detected, which periodically brought the neuron over threshold to fire overshooting action potentials. $B$, This activity was completely abolished by addition of $0.1 \mu \mathrm{M}$ TTX to the perfusate.

We wished to know whether the number of GABA-positive neurons in these cultures is influenced by spontaneous activity. The percentage of neurons in culture that were GABA-positive was determined by double-labeling cultures against both the neuronal marker MAP2 and GABA (Fig. 2A,B). All GABA-positive neurons were MAP2-positive. The GABA-positive neurons had a variety of morphologies, including bipolar and multipolar, associated with GABAergic interneurons in vivo (Fig. $2 C, D$ ). In control cultures, $29.6 \pm 1.0 \%$ of the neurons were found to be GABApositive $(n=26)$. This percentage is within the range reported for visual cortex of primate, rat, and cat (Gabbott and Somogyi, 1986; Hendry and Jones, 1986; Benevento et al., 1995) and in dissociated cortical cultures (Alho et al., 1988; Gotz and Boltz, 1994). Between 7 and $14 \mathrm{~d}$ in vitro, there was no significant effect of time in culture on the percentage of GABA-positive neurons; therefore, data from these ages were combined.

Treatment of cultures for $2 \mathrm{~d}$ with TTX reduced the percentage of GABA-positive neurons to $19.2 \pm 0.9 \%$. This represents a decrease to $64.9 \pm 3.0 \%$ of control values (Fig. $3 A, T T X)(n=14$, TTX significantly different from control, Student's $t$ test, $p<$ 0.01). This effect is similar in magnitude to the effect of visual deprivation on the number of GABA-positive neurons in rat and primate visual cortex (a reduction to $\sim 70 \%$ of control values) (Hendry and Jones, 1986; Benevento et al., 1995). There was no effect of TTX treatment on neuronal survival (Table 1). Measurement of input resistances and resting membrane potentials of bipolar interneurons (a subset of interneurons that can be readily morphologically identified) revealed no effect of TTX treatment on these parameters (Table 2). Control experiments showed that TTX completely blocked spike generation for the duration of the treatment (data not shown).

BDNF prevents the activity-dependent reduction in the number of GABA-positive neurons

BDNF levels in visual cortex can be rapidly modulated by activity (Castrén et al., 1992) and can regulate the phenotype of GABAergic interneurons in hippocampus, mesencephalon, striatum, and cortex (Hyman et al., 1994; Mizuno et al., 1994; Marty et al., 1996a,b). This suggests that visual input may regulate cortical GABA expression through the activity-dependent regulation of
BDNF. If the reduction in the number of GABA-positive neurons in our visual cortical cultures produced by activity blockade is the consequence of reduced endogenous production of BDNF, then exogenous BDNF should prevent this reduction. We tested this possibility by applying TTX for $2 \mathrm{~d}$ in the presence of $25 \mathrm{ng} / \mathrm{ml}$ BDNF. Under these conditions, there was no reduction in the percentage of GABA-positive neurons over control values (Fig. $3 A, T T X+B D N F)(n=8)$. TTX + BDNF was significantly different from TTX alone ( $p<0.01$, Student's $t$ test) and was not different from control. BDNF significantly reduced the effects of TTX on GABA levels at concentrations as low as $1 \mathrm{ng} / \mathrm{ml}$ (the lowest concentration tested) and was saturating at concentrations between 5 and $10 \mathrm{ng} / \mathrm{ml}$ (Fig. 3B). Interestingly, when BDNF (25 $\mathrm{ng} / \mathrm{ml}$ ) was applied for $2 \mathrm{~d}$ in the presence of spontaneous activity, there was no effect on the percentage of GABA-positive neurons (Fig. $3 A, B D N F)(n=9)$.

Like BDNF, NT3 is expressed in visual cortex during prenatal and early postnatal development, but levels peak at approximately $\mathrm{P} 0$ and then decrease steadily, whereas BDNF levels increase steadily during the first three postnatal weeks (Maisonpierre et al., 1990; Schoups et al., 1995). NGF and its receptor TrkA are present only at very low levels in cortex (Maisonpierre et al., 1990; Allendoerfer et al., 1994; Schoups et al., 1995). Neither NGF nor NT3 was effective at preventing the reduction in GABA produced by TTX. Incubation for $2 \mathrm{~d}$ with TTX and $50 \mathrm{ng} / \mathrm{ml} \mathrm{NGF}$ reduced the percentage of GABA-positive neurons to $68.2 \pm 3.4 \%$ of control values (Fig. $3 A, T T X+N G F)(n=6)$. Incubation with TTX and $25 \mathrm{ng} / \mathrm{ml} \mathrm{NT3}$ produced a similar reduction, to $71.6 \pm$ $1.6 \%$ of control values (Fig. $3 A, T T X+N T 3)(n=5)$. These treatments were not significantly different from TTX alone. None of these manipulations significantly influenced neuronal survival (Table 1).

\section{Blockade of neurotrophin receptor signaling reduces the number of GABA-positive neurons}

The above data suggest that activity blockade is reducing the percentage of GABA-positive neurons in culture by reducing the production of BDNF. This raised the question of whether blocking the action of endogenous neurotrophins might influence the expression of GABA in these cortical cultures. The neurotrophins act through the Trk tyrosine kinase receptors TrkA (NGF), TrkB (BDNF), and TrkC (NT3) (for review, see Heumann, 1994). This family of receptors is blocked by the compound $\mathrm{K} 252 \mathrm{a}$, which prevents autophosphorylation of the tyrosine kinase domain of the receptors. At concentrations of $200 \mathrm{~nm}$ or less, $\mathrm{K} 252 \mathrm{a}$ is a specific inhibitor of Trk receptors and blocks TrkA, TrkB, and TrkC with approximately equal efficacy, but leaving other tyrosine kinase signaling pathways, as well as protein kinase $\mathrm{C}$ pathways, intact (Kiozumi et al., 1988; Berg et al., 1992; Nye et al., 1992; Tapley et al., 1992).

To test whether endogenous neurotrophin signaling influences GABA expression, cultures were incubated for $2 \mathrm{~d}$ with various concentrations of K252a. Concentrations of K252a as low as $10 \mathrm{~nm}$ produced a decrease in the percentage of GABA-positive neurons $(n=3)$, and at concentrations of $50 \mathrm{~nm}$ and above, K252a significantly reduced the percentage of GABA-positive neurons to $65.3 \pm 1.8$ and $62.1 \pm 3.4 \%$ of control, respectively (Fig. 4$)$. The reduction produced by $50 \mathrm{~nm}(n=3)$ and $200 \mathrm{~nm}(n=9) \mathrm{K} 252 \mathrm{a}$ was comparable with that produced by blockade of activity with TTX (see Fig. 3A). In the presence of 200 nм K252a, BDNF did not prevent the TTX-induced reduction in the percentage of GABA-positive neurons (Fig. 4, TTX $+B D N F+K 252 a)(n=3)$, 

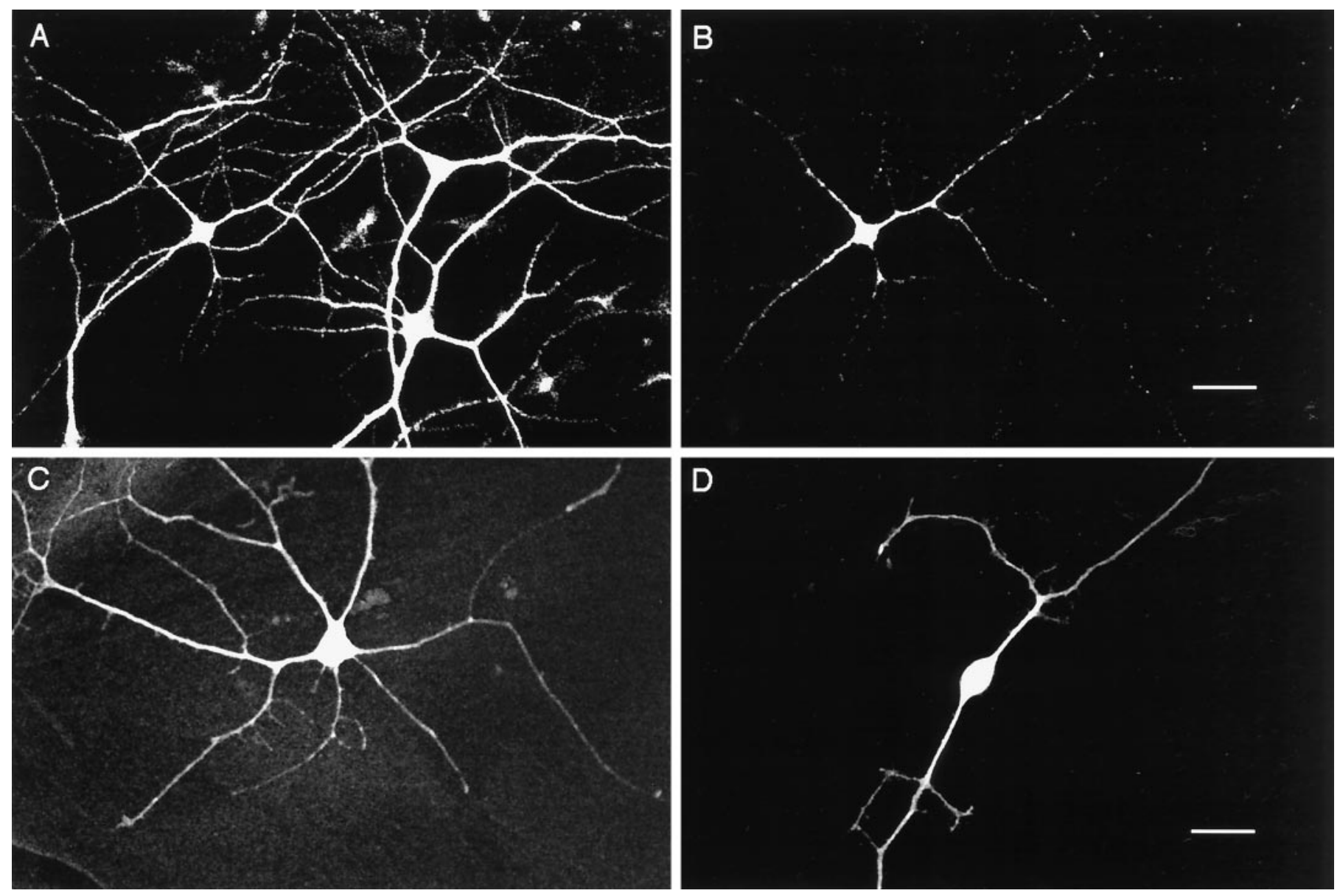

Figure 2. GABA immunoreactivity in cortical cultures. A, MAP2-positive neurons from cortical cultures after $7 \mathrm{~d}$ in vitro, viewed with fluorescein filters. $B$, Same field of view as in $A$, using rhodamine filters to show GABA-positive neurons. $C$, GABA-positive multipolar neuron. $D$, GABA-positive bipolar neuron. Scale bars: $A, B, 10 \mu \mathrm{m} ; C, D, 25 \mu \mathrm{m}$.

Table 1. Effects of culture conditions on neuronal survival

\begin{tabular}{lc} 
Condition & $\begin{array}{l}\text { Neuronal density } \\
(\% \text { control })\end{array}$ \\
\hline TTX (14) & $102 \pm 13$ \\
TTX + BDNF (11) & $127 \pm 16$ \\
TTX + NGF (6) & $130 \pm 13$ \\
BDNF (9) & $98 \pm 14$ \\
K252a (9) & $102 \pm 8$ \\
TTX + WASH (6) & $97 \pm 16$ \\
TTX + WASH + BDNF (3) & $96 \pm 6$ \\
\hline
\end{tabular}

Neuronal density in cultures grown for $2 \mathrm{~d}$ under the different experimental conditions. Drug dosages are as reported in Results. Values are mean \pm SEM for the number of cultures indicated in parentheses.

indicating that K252a was effectively blocking Trk receptor signaling at this concentration. K252a treatment had no influence on neuronal survival at any of the concentrations tested (Table 1). These data suggest that endogenous neurotrophin signaling through Trk receptors is regulating GABA expression in cortical interneurons. These data do not allow us to distinguish between signaling through the different Trk receptors, but given that neither NT3 nor NGF has any effect on GABA expression in this system, the most likely possibility is that this effect is mediated by endogenous release of BDNF.

\begin{tabular}{lll}
\hline \multicolumn{3}{l}{ Table 2. Electrical properties of cortical neurons in culture } \\
Pyramidal morphology & $\begin{array}{l}\text { Membrane } \\
\text { potential }(\mathrm{mV})\end{array}$ & $\begin{array}{l}\text { Input resistance } \\
(\mathrm{G} \Omega)\end{array}$ \\
\hline Control (13) & $-63.4 \pm 1.3$ & $0.97 \pm 0.05$ \\
TTX (14) & $-63.6 \pm 0.8$ & $1.02 \pm 0.02$ \\
TTX + BDNF (10) & $-62.4 \pm 1.5$ & $0.99 \pm 0.06$ \\
Bipolar morphology & & \\
Control (11) & $-62.6 \pm 1.1$ & $1.11 \pm 0.05$ \\
TTX (11) & $-60.9 \pm 1.5$ & $1.19 \pm 0.07$ \\
\hline
\end{tabular}

Resting potentials and input resistances of pyramidal and bipolar neurons grown for $2 \mathrm{~d}$ under the different experimental conditions. Values were measured under conditions where synaptic transmission was blocked with CNQX and bicuculline. Values are mean \pm SEM for the number of neurons indicated in parentheses.

\section{The activity-dependent reduction in the number of GABA-positive neurons is reversible}

Studies on the role of visual input and neuronal activity on GABA expression in visual cortex have suggested that decreased activity acts to lower GABA levels in surviving neurons, rather than to decrease interneuronal survival (Hendry and Jones, 1986; Benson et al., 1991). These studies have suggested a model in which activity can continuously modulate the amount of GABA produced by cortical interneurons. This model predicts that the activity-dependent reduction in the number of GABA-positive 
A

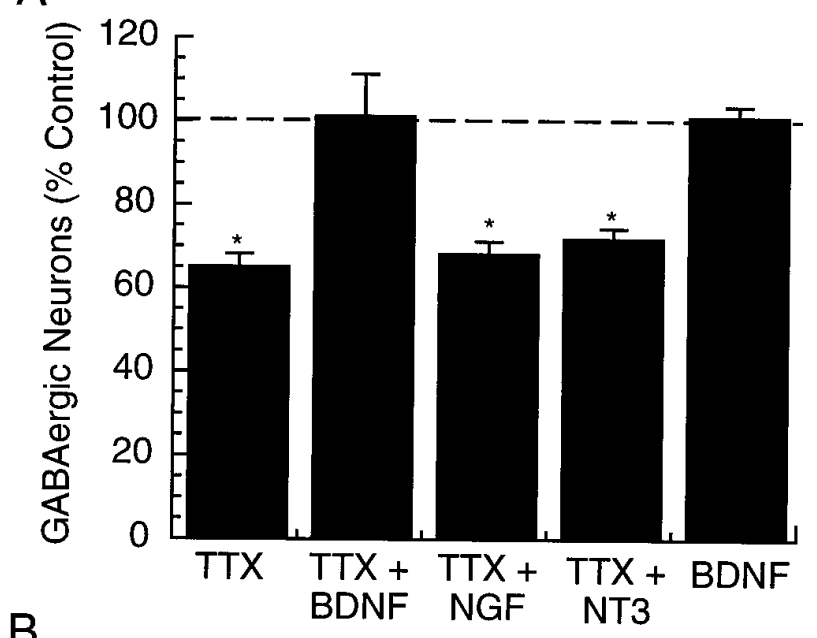

$\mathrm{B}$

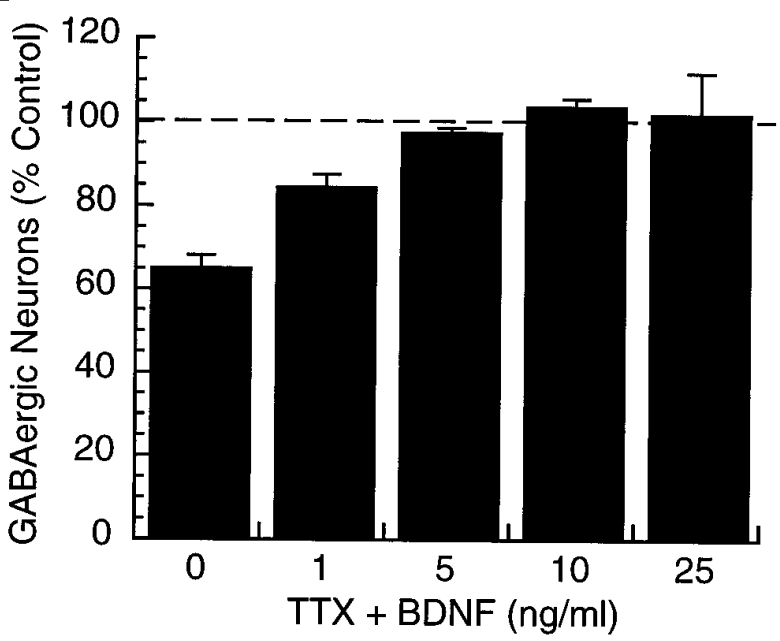

Figure 3. The effects of activity blockade and neurotrophins on the percentage of GABA-positive neurons in cortical cultures. $A$, Cultures were treated with $0.1 \mu \mathrm{M}$ TTX, either alone $(T T X)$ or in the presence of $25 \mathrm{ng} / \mathrm{ml}$ BDNF $(T T X+B D N F), 50 \mathrm{ng} / \mathrm{ml} \mathrm{NGF}(T T X+N G F)$, or 25 $\mathrm{ng} / \mathrm{ml} \mathrm{NT3}(T T X+N T 3)$ for $2 \mathrm{~d}$; * significantly different from control, $p<$ 0.01 . $B$, The effects of different doses of BDNF on the ability of TTX to reduce the percentage of GABA-positive neurons were determined. TTX $(0.1 \mu \mathrm{M})$ was applied in the presence of the indicated concentration of BDNF for $2 \mathrm{~d}$ and the percentage of GABA-positive neurons determined. For each condition in $A$ and $B$, the ratio of GABA-positive to GABAnegative neurons was determined, and these values are expressed as a percent of the values obtained for control cultures (control $=100 \%$, dashed line).

neurons in visual cortex should be reversible on reinstatement of activity, but this has not been tested.

To address this issue, activity was blocked for $2 \mathrm{~d}$ with TTX, then TTX was washed out and the cultures incubated for two additional days in normal medium. Control cultures were washed similarly and fixed and processed in parallel. Control experiments indicated that $2 \mathrm{~d}$ after TTX washout, spike generation was normal (data not shown). GABA levels were partially restored $2 \mathrm{~d}$ after TTX washout, to $82.7 \pm 3.0 \%$ of control values (Fig. 5$)(n=$ 6 , wash significantly different from TTX alone, $p<0.05$, Student's $t$ test). When BDNF $(25 \mathrm{ng} / \mathrm{ml})$ was added immediately after TTX washout, GABA levels were completely restored to control levels after $2 \mathrm{~d}(106.0 \pm 8.7 \%$ of control, $n=3)$ (Fig. 5). These data

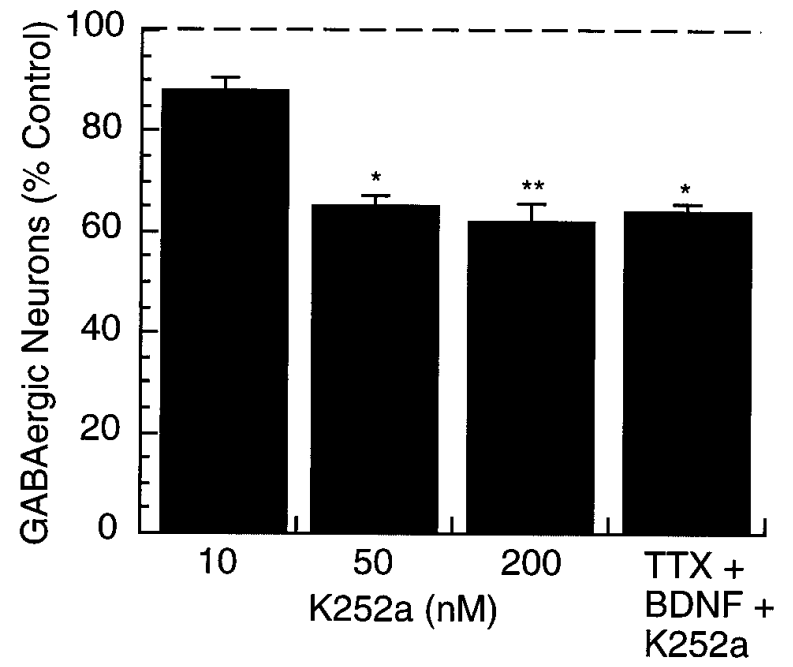

Figure 4. The effects of Trk receptor blockade on the percentage of GABA-positive neurons. K252a, a blocker of Trk receptor signaling, was applied for $2 \mathrm{~d}$ at the indicated concentration $(10,50$, or $200 \mathrm{nM})$ and the percentage of GABA-positive neurons determined. The effect of BDNF $(25 \mathrm{ng} / \mathrm{ml})$ and TTX $(0.1 \mu \mathrm{M})$ in the presence of K252a $(200 \mathrm{nM})$ for $2 \mathrm{~d}$ was also determined $(T T X+B D N F+K 252 a)$. Numbers are expressed as a percentage of the value obtained for control cultures (control $=100 \%$, dashed line); *significantly different from control, $p<0.05$; **significantly different from control, $p<0.001$.

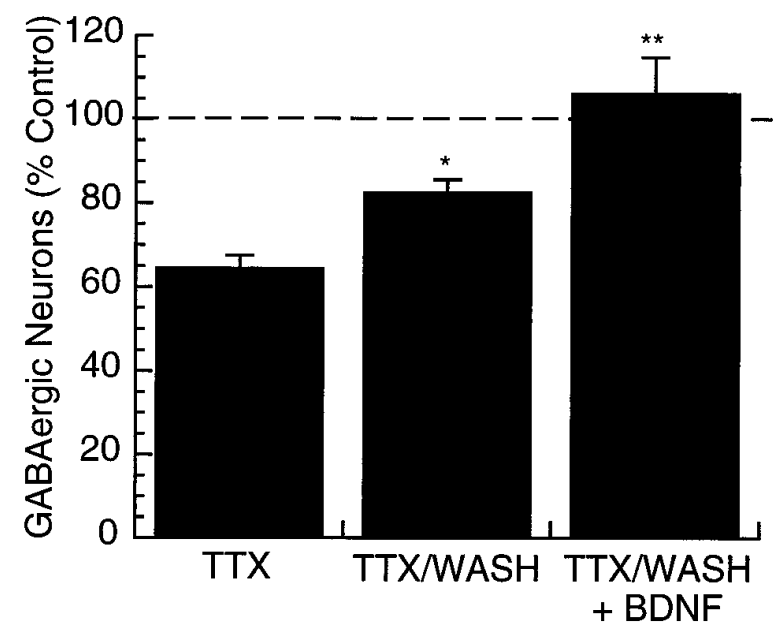

Figure 5. The activity-dependent reduction in the percentage of GABApositive neurons is reversible. Cultures were treated with TTX $(0.1 \mu \mathrm{M})$ for $2 \mathrm{~d}$. Cultures were then fixed, processed, and counted immediately $(T T X)$; washed for $2 \mathrm{~d}$ before fixation $(T T X / W A S H)$; or washed and BDNF $(25 \mathrm{ng} / \mathrm{ml})$ added for $2 \mathrm{~d}$ before fixation. Numbers are expressed as a percentage of the value obtained for control cultures (control $=100 \%$, dashed line); *significantly different from control, $p<0.05 ;{ }^{*}$ significantly different from control, $p<0.01$.

indicate that the reduction in the percentage of GABA-positive neurons produced by activity blockade is fully reversible and that the degree of reversal can be influenced by exogenous BDNF.

\section{Activity blockade reduces inhibition onto cortical pyramidal neurons}

Although decreased activity is known to decrease the number of GABA-immunopositive neurons in visual cortex (Hendry and 
A
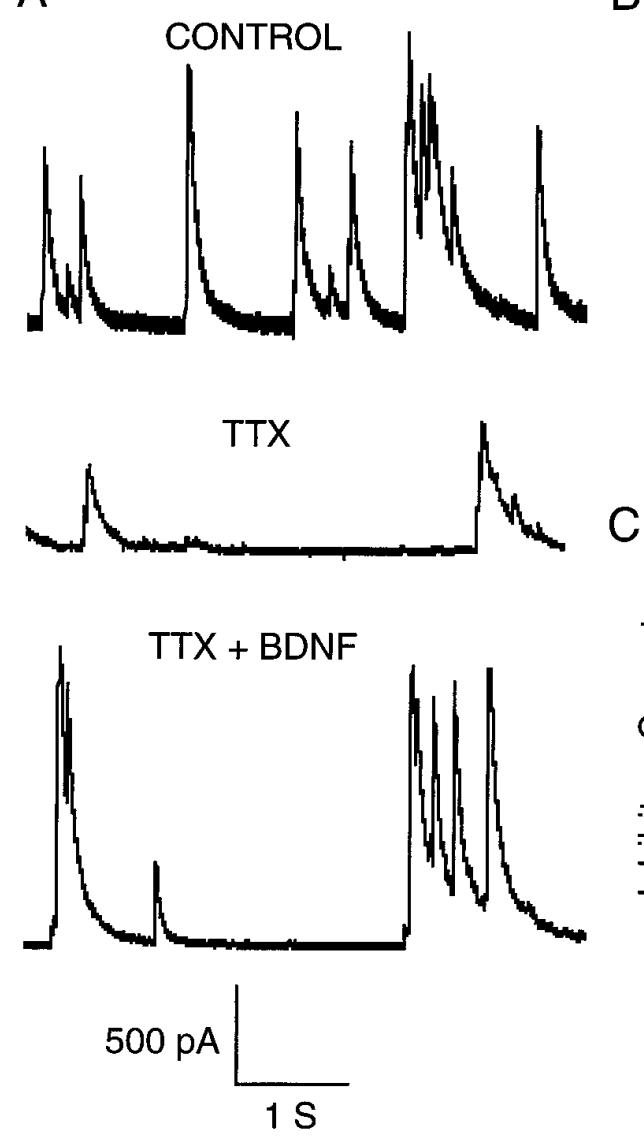

B
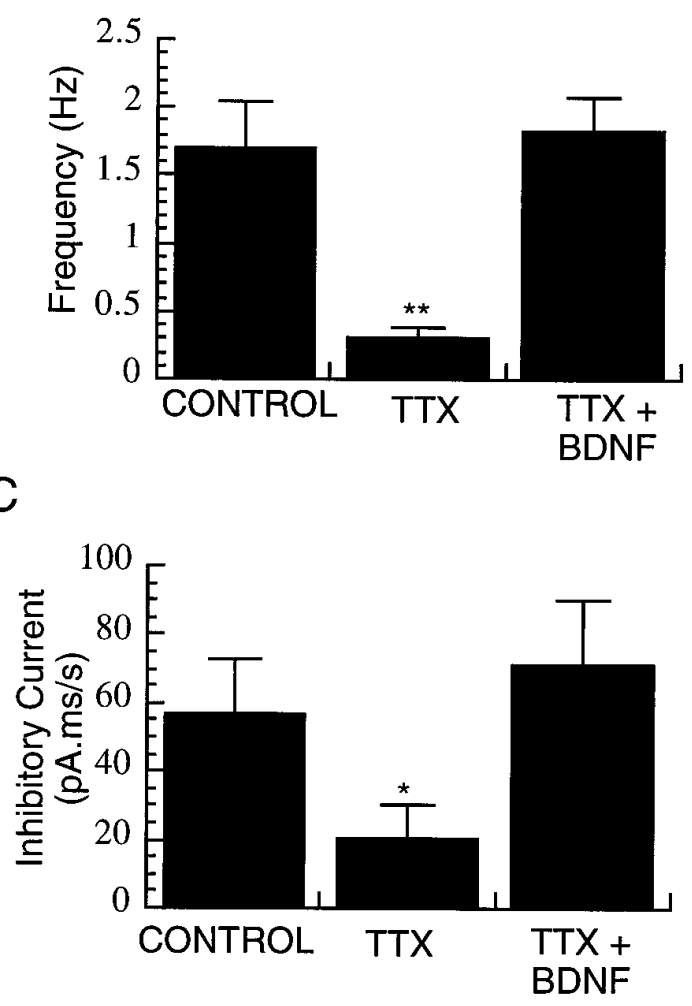

Figure 6. Activity blockade reduces inhibition onto pyramidal neurons. $A$, Representative voltage-clamp recordings of spontaneous IPSCs from pyramidal neurons grown in control medium $(C O N T R O L)$ in medium supplemented with $0.1 \mu \mathrm{M}$ TTX for $2 \mathrm{~d}(T T X)$ or in medium supplemented with TTX and BDNF (25 ng $/ \mathrm{ml})$ for $2 \mathrm{~d}$. $B$, Frequency of spontaneous IPSCs from pyramidal neurons grown under the conditions indicated in $A$ ( $n=8$ neurons in each condition). $C$, Total inhibitory current integrated over time, from the same population as in $B$; *significantly different from control (Student's $t$ test, $p<$ $0.04) ; *{ }^{*} p<0.01$. animals that correlate with decreases in the number of GABApositive neurons (Benevento et al., 1995). We asked whether the reduction in inhibition onto pyramidal neurons produced by activity blockade in our cortical cultures was correlated with an increase in pyramidal neuron firing rates. Whole-cell recordings were obtained from pyramidal neurons from control cultures or from cultures treated for $2 \mathrm{~d}$ with TTX after at least $0.5 \mathrm{hr}$ washout of the TTX. TTX treatment was found to increase pyramidal neuron firing rates by more than an order of magnitude, from $1.6 \pm 0.3 \mathrm{spikes} / \mathrm{min}(n=29)$ to $16.5 \pm 3.5 \mathrm{spikes} / \mathrm{min}$ (Fig. 7) $(n=23)$. Co-incubation with TTX and BDNF $(25 \mathrm{ng} / \mathrm{ml})$, which prevents the reduction in inhibition, also prevents the increase in pyramidal neuron firing rates (spike frequency was $1.4 \pm 0.5, n=13$ ). In contrast, NT3 and NGF, which do not prevent the reduction in the number of GABA-positive neurons, also do not prevent the increase in pyramidal neuron firing rates (Fig. 7) ( $n=14$ and 11, respectively). No differences were found in input resistances or resting membrane potentials from neurons grown under the different experimental conditions (Table 2). These data indicate that activity can regulate pyramidal neuron firing rates and suggest that one mechanism of this regulation is the BDNF-dependent modulation of inhibition.

\section{DISCUSSION}

Sensory deprivation leads to a decrease in the number of GABAimmunopositive neurons in both somatosensory and visual cortex (Hendry and Jones, 1986; Warren et al., 1989; Kossut et al., 1991; Benevento et al., 1995). This decrease in GABA immunoreactivity appears to be a general response of cortical circuits to a reduction 
A
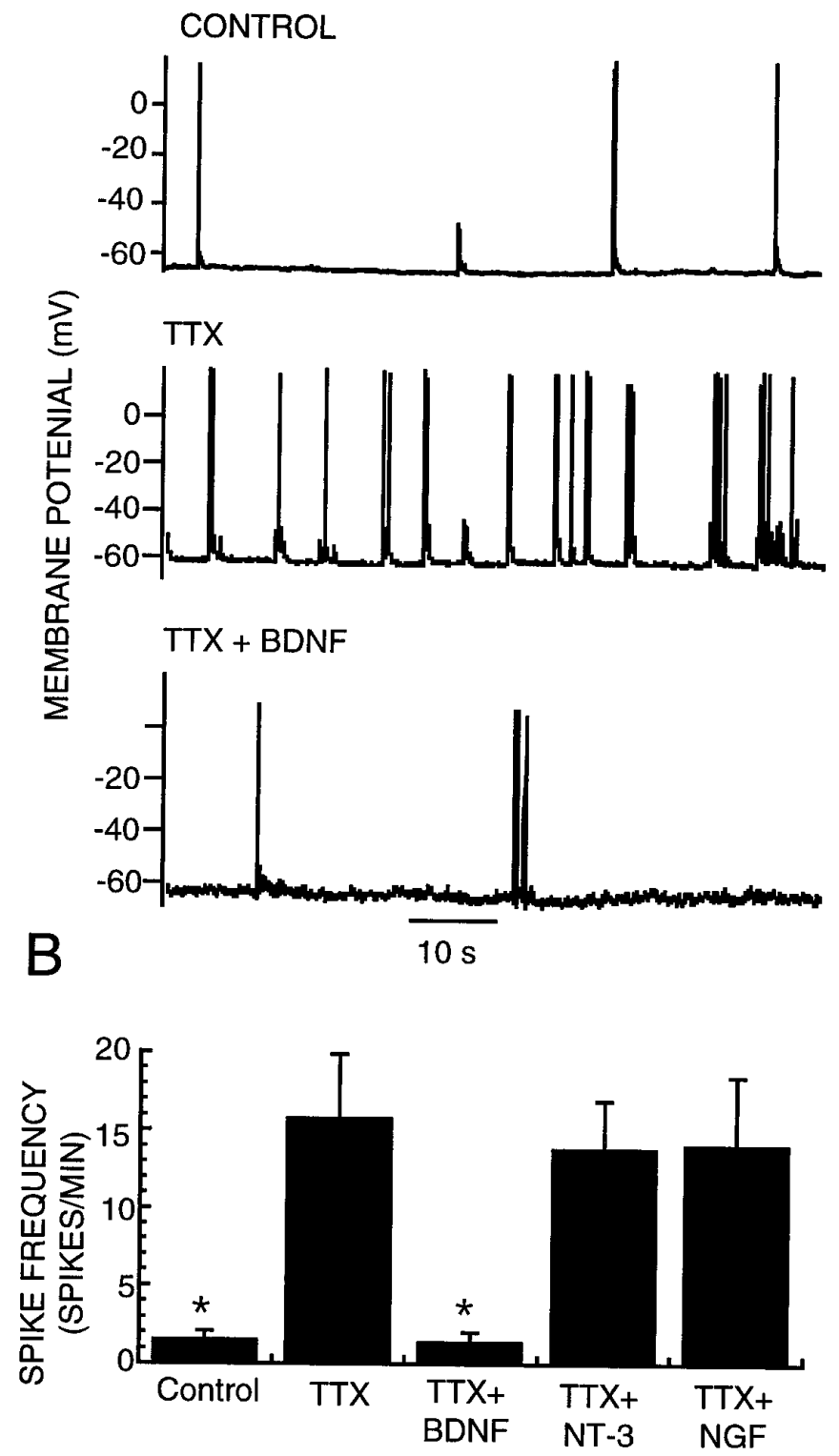

Figure 7. Activity blockade increases pyramidal neuron firing rates. $A$, Representative current-clamp recordings of spontaneous firing from pyramidal neurons grown in control medium $(C O N T R O L)$, in medium supplemented with $0.1 \mu \mathrm{M}$ TTX for $2 \mathrm{~d}(T T X)$, or in medium supplemented with TTX and BDNF $(25 \mathrm{ng} / \mathrm{ml})$ for $2 \mathrm{~d}(T T X+B D N F)$. B, Average spike frequency of pyramidal neurons grown for $2 \mathrm{~d}$ under the conditions described in $A$ or in TTX $+50 \mathrm{ng} / \mathrm{ml} \mathrm{NGF}(T T X+N G F)$ or TTX $+25 \mathrm{ng} / \mathrm{ml} \mathrm{NT3}(T T X+N T 3)$; *significantly different from TTX (Student's $t$ test, $p<0.01$ ).

in excitatory drive and suggests that activity levels can regulate the strength of cortical inhibition in both developing and adult animals. In this study, we use an in vitro model system to ask whether activity-dependent changes in GABA expression lead to a functional change in cortical inhibition and to address the mechanism by which this process occurs.

We show that the number of GABA-positive neurons can be decreased in postnatal cortical cultures by blockade of spontaneous activity and that this reduction can be reversed on reinstatement of activity. The magnitude of this effect, an approximate
$30 \%$ reduction in the number of GABA-positive neurons, is similar to that observed in intact visual cortex after blockade of visual input (Hendry and Jones, 1986; Benevento et al., 1995). Further, we show that the decrease in GABA immunoreactivity can be blocked by BDNF, but not NGF or NT3, and can be mimicked by blockade of Trk receptor signaling with K252a. Finally, we show that the reduction in GABA is correlated with a decrease in the amount and frequency of inhibitory currents onto cortical pyramidal neurons and an increase in pyramidal neuron firing rates. These data suggest that activity continuously adjusts cortical excitability through the BDNF-dependent regulation of inhibition.

All of the effects of activity blockade on our cortical cultures, including the reductions in the number of GABA-immunopositive neurons, the reduction in inhibition, and the increase in pyramidal neuron firing rates, were blocked by co-application of exogenous BDNF. This suggests that activity blockade is exerting these effects through a reduction in endogenous BDNF production. A similar model has been proposed for the activity-dependent regulation of peptide expression in hippocampal interneurons (Marty et al., 1996a). Interestingly, BDNF had no significant effect on any of the parameters measured when activity was intact. BDNF alone did produce a small increase in the frequency of inhibitory currents over control values, but these effects were not statistically significant (Fig. 6), and BDNF alone had no effect on the number of GABA-immunopositive neurons (Fig. 3) or on pyramidal neuron firing rates. The failure of exogenous BDNF to significantly influence these parameters with activity intact suggests that under control conditions, endogenous production of BDNF is close to saturating. If so, then exogenous BDNF would only have an effect under conditions in which endogenous production was reduced, such as activity blockade. Although cortical neuronal cultures are known to express BDNF in an activity-dependent manner, the concentration of this neurotrophin in cortical cultures is unknown (Ghosh et al., 1994).

The effects of BDNF reported here are expressed in the absence of neuronal activity. This is in contrast to several other reported effects of BDNF on cortical neurons, including effects on survival and on dendritic growth (Ghosh et al., 1994; McAllister et al., 1996), although BDNF has been shown to regulate peptide expression in hippocampal interneurons in the absence of spiking activity (Marty et al., 1996b). This suggests that there are heterogeneous mechanisms by which BDNF influences different cortical neuronal properties, some of which require conjoint activity in target neurons and some of which do not. The data reported here show that BDNF regulates inhibition onto cortical pyramidal neurons in the absence of spike generation and spike-mediated synaptic activity. One of the likely functions of this activitydependent regulation of inhibition is to globally reduce inhibition when activity levels fall too low. To participate in this process, BDNF must be capable of regulating inhibition during periods of absent or very low activity.

BDNF can promote survival of several populations of cultured central neurons, including retinal ganglion cells (Myer-Franke et al., 1995) and largely pure embryonic cortical neurons (Ghosh et al., 1994), as well as some populations of sensory and autonomic neurons (Buj-Bello et al., 1995). In our postnatal cortical cultures, we observed no effect of BDNF on survival over a $2 \mathrm{~d}$ period. Additionally, blockade of Trk receptors with K252a for $2 \mathrm{~d}$ produced no decrease in neuronal survival. K252a was able to completely block the effects of exogenous BDNF, indicating that it was effectively blocking Trk receptors. K252a is effective at reducing 
neurotrophin-mediated survival in other systems (Doherty et al., 1989). The failure of K252a to reduce survival in our cultures suggests that under the culture conditions used, in which cortical neurons were co-cultured with cortical astrocytes, survival of postnatal cortical neurons is independent of Trk receptormediated signaling pathways. There is evidence that multiple survival signals converge on some populations of central neurons so that dependence on any one factor is not absolute (MyerFranke et al., 1995).

Several observations suggest that activity blockade is reducing GABA expression within surviving interneurons below our ability to detect rather than reducing interneuronal survival. First, total neuronal survival was not compromised by activity blockade or by any of the manipulations performed in this study. Second, the decrease in GABA-positive neurons was completely reversible. This suggests that a surviving pool of interneurons can be reinduced to express GABA after reinstatement of activity. The alternative possibility is that reversal of activity blockade is inducing a new population of GABAergic neurons to differentiate from a population of neuronal precursor cells present in culture. This is unlikely because cultured embryonic cortical neurons are already postmitotic (Ghosh et al., 1994), and our postnatal cultures are made subsequent to the period of cortical neuronal birth.

The reduction in the number of GABA-positive neurons produced by activity blockade is correlated with a reduction in functional inhibition onto pyramidal neurons. Both the frequency and the magnitude of spontaneous IPSCs were dramatically reduced. An interesting question is whether the change in IPSC frequency is accompanied by a change in the number of synaptic contacts between inhibitory interneurons and pyramidal neurons. Blockade of activity with TTX has been reported to reduce inhibitory synaptogenesis onto cerebellar Purkinje neurons in organotypic cultures (Seil and Drake-Baumann, 1994). An intriguing possibility is that transmitter expression, inhibitory synaptic strength, and the number of synaptic contacts in our cortical cultures are regulated in tandem by ongoing electrical activity.

The decreased inhibition produced by activity blockade is correlated with a dramatic increase in pyramidal neuron firing rates. Consistent with this, electrophysiological recordings from visual cortex of dark-reared rats show an increase in spontaneous activity relative to control animals that correlate with a decrease in the number of GABA-positive neurons (Benevento et al., 1995). In addition, chronic treatments that block spontaneous activity, such as TTX, have been reported to produce hyperexcitability in cortical cultures when washed out, as would be expected for manipulations that reduced inhibition (Ramakers et al., 1990). Activity is known to regulate a number of properties of neurons and circuits, including the expression of conductances that influence intrinsic excitability (Turrigiano et al., 1994, 1995). Although the reduction in inhibition reported here is likely to contribute substantially to the increase in pyramidal neuron firing rates, other factors are also likely to contribute. In addition to reducing inhibition, activity blockade increases the amplitude of miniature excitatory synaptic currents onto pyramidal neurons, suggesting that inhibition and excitation are reciprocally regulated by activity (Turrigiano et al., 1996). Interestingly, BDNF is able to completely prevent the effects of activity blockade on pyramidal neuron firing rates, suggesting that any factors contributing to this change in firing rate are being conjointly regulated by BDNF.

The data we have presented suggest that activity is continuously modulating the amount of inhibition in cortical circuits and that this results in an adjustment in pyramidal neuron firing rates. Our data suggest that this activity-dependent regulation of circuit excitability is mediated through the activity-dependent regulation of BDNF. This in turn suggests that an important function for this neurotrophin is in the control of cortical excitability.

\section{REFERENCES}

Alho H, Ferrarese C, Vicini S, Vaccarino F (1988) Subsets of GABAergic neurons in dissociated cell cultures of neonatal rat cerebral cortex show co-localization with specific modulator peptides. Brain Res 467:193-204.

Allendoerfer KL, Cabelli RJ, Escandon E, Kaplan DR, Nikolics E, Shatz CJ (1994) Regulation of neurotrophin receptors during the maturation of the mammalian visual system. J Neurosci 14:1795-1811.

Benevento LA, Bakkum BW, Cohen RS (1995) Gamma-aminobutyric acid and somatostatin immunoreactivity in the visual cortex of normal and dark-reared rats. Brain Res 689:172-182.

Benson DL, Isackson PJ, Gall CM, Jones EG (1991) Differential effects of monocular deprivation on glutamic acid decarboxylase and type II calcium-calmodulin-dependent protein kinase gene expression in the adult monkey visual cortex. J Neurosci 11:31-47.

Berg M, Sternberg D, Parada L, Choa M (1992) K252a inhibits nerve growth factor-induced Trk proto-oncogene tyrosine phosphorylation and kinase activity. J Biol Chem 267:13-16.

Buj-Bello A, Buchman VL, Horton A, Rosenthal A, Davies AM (1995) GDNF is an age-specific survival factor for sensory and autonomic neurons. Neuron 15:821-828.

Cabelli RJ, Hohn A, Shatz CJ (1995) Inhibition of ocular dominance column formation by infusion of NT-4/5 or BDNF. Science 267 : 1662-1666.

Castrén E, Zafra F, Thoenen H, Lindholm D (1992) Light regulates expression of brain-derived neurotrophic factor mRNA in rat visual cortex. Proc Natl Acad Sci USA 89:9444-9448.

Chagnac-Amitai Y, Conners BW (1989) Synchronized excitation and inhibition driven by intrinsically bursting neurons in neocortex. J Neurophysiol 62:1149-1162.

Doherty P, Walsh FS (1989) K252a specifically inhibits the survival and morphological differentiation of NGF-dependent neurons in primary cultures of human dorsal root ganglion. Neurosci Lett 96:1-6.

Gabbott PL, Somogyi P (1986) Quantitative distribution of GABAimmunoreactive neurons in the visual cortex of the cat. Exp Brain Res 61:323-331.

Ghosh A, Carnahan J, Greenberg ME (1994) Requirement for BDNF in activity-dependent survival of cortical neurons. Science 263:1618-1623.

Gotz M, Bolz J (1994) Differentiation of transmitter phenotypes in rat cerebral cortex. Eur J Neurosci 6:18-32.

Hendry SHC, Jones EG (1986) Reduction in number of immunostained GABAergic neurones in deprived-eye dominance columns of monkey area 17. Nature 320:750-753.

Heumann R (1994) Neurotrophin signalling. Curr Opin Neurobiol 4:668-679.

Huettner JE, Baughman RW (1986) Primary cultures of identified neurons from the visual cortex of postnatal rats. J Neurosci 6:3044-3060.

Huettner JE, Baughman RW (1988) The pharmacology of synapses formed by identified corticocollicular neurons in primary cultures of rat visual cortex. J Neurosci 8:160-175.

Hyman C, Juhasz M, Jackson C Wright P, Ip NY, Lindsay RM (1994) Overlapping and distinct actions of the neurotrophins BDNF, NT-3, and NT-4/5 on cultured dopaminergic and GABAergic neurons of the ventral mesancephalon. J Neurosci 14:335-347.

Isackson PJ, Huntsman MM, Murray KD, Gall CM (1991) BDNF mRNA expression is increased in adult rat forebrain after limbic seizures: temporal patterns of induction distinct from NGF. Neuron 6:937-948.

Koizumi S, Contreras ML, Matsuda Y, Hama T, Lazarovici P, Curoff G (1988) K-252a: a specific inhibitor or the action of nerve growth factor on PC12 cells. J Neurosci 8:715-721.

Kossut M, Stewart MG, Siucinska E, Bourne RC, Gabbott PLA (1991) Loss of gama-aminobytyric acid (GABA). Immunoreactivity from mouse first somatosensory (SI) cortex following neonatal, but not adult, denervation. Brain Res 538:165-170.

Kreigstein AR, Suppes T, Prince DA (1987) Cellular and synaptic physiology and epileptogenesis of developing rat neocortical neurons in vitro. Dev Brain Res 34:161-171. 
Lindsay RM, Wiegand SJ, Altar CA, Sistefano PS (1994) Neurotrophic factors: from molecule to man. Trends Neurosci 17:182-190.

Maisonpierre PC, Belluscio L, Friedman B, Alderson RF, Wiegand SJ, Furth ME, Lindsay RM, Yancopoulos GD (1990) NT-3, BDNF, and NGF in the developing rat nervous system: parallel as well as reciprocal patterns of expression. Neuron 5:501-509.

Marty S, Berninger B, Carroll P, Thoenen H (1996a) GABAergic stimulation regulates the phenotype of hippocampal interneurons through the regulation of brain-derived neurotrophic factor. Neuron 16:565-570.

Marty S, Carroll P, Cellerino A, Castrén E, Staiger V, Thoenen H, Lindholm D (1996b) Brain-derived neurotrophic factor promotes the differentiation of various hippocampal nonpyramidal neurons, including Cajal-Retzius cells, in organotypic slice cultures. J Neurosci 16:675-687.

McAllister AK, Lo DC, Katz LC (1995) Neurotrophins regulate dendritic growth in developing visual cortex. Neuron 15:791-803.

McAllister AK, Katz LC, Lo DC (1996) Neurotrophin regulation of cortical dendritic growth requires activity. Neuron 17:1057-1064.

Mizuno K, Carhahan J, Nawa H (1994) Brain-derived neurotrophic factor promotes differentiation of striatal GABAergic neurons. Dev Biol 165:243-256.

Myer-Franke A, Kaplan MR, Pfrieger FW, Barres BA (1995) Characterization of the signaling interactions that promote the survival and growth of developing retinal ganglion cells in culture. Neuron 15:805-819.

Nye S, Squinto S, Glass D, Stitt T, Hantzopoulos G (1992) K252a and staurosporine selectively block autophosphorylation of neurotrophin receptors and neurotrophin-mediated responses. Mol Biol Cell 3:677-686.

Ramakers GJA, Corner MA, Habets AM (1990) Development in the absence of spontaneous bioelectric activity results in increased stereo- typed burst firing in cultures of dissociated cerebral cortex. Exp Brain Res 79:157-166.

Schoups AA, Elliott RC, Friedman WJ, Black IB (1995) NGF and BDNF are differentially modulated by visual experience in the developing geniculocortical pathway. Brain Res 86:326-334.

Seil FJ, Drake-Baumann R (1994) Reduced cortical inhibitory synaptic in organotypic cerebellar cultures developing in the absence of neuronal activity. J Comp Neurol 342:366-377.

Tapley P, Lamballe F, Barbacid M (1992) K252a is a selective inhibitory of the tyrosine protein kinase activity of the Trk family of oncogenes and neurotrophin receptors. Oncogene 7:371-381.

Turrigiano GG, Abbott LF, Marder E (1994) Activity-dependent changes in the intrinsic properties of cultured neurons. Science 264: 974-976.

Turrigiano GG, Le Masson G, Marder E (1995) Selective regulation of current densities underlies spontaneous changes in the activity of cultured neurons. J Neurosci 15:3640-6552.

Turrigiano GG, Leslie KR, Desai N, Nelson SB (1996) Activitydependent regulation of quantal amplitude in visual cortical cultures. Soc Neurosci Abstr 22:275.

Ventimiglia R, Mather PE, Jones BE, Lindsay RM (1995) The neurotrophins BDNF, NT-3, and NT-4/5 promote survival and morphological and biochemical differentiation of striatal neurons in vitro. Eur J Neurosci 7:213-222.

Warren R, Trembray N, Dykes RW (1989) Quantitative study of glutamic acid decarboxylase-immunoreactive neurons and cytochrome oxidase activity in normal and partially deafferented rat hindlimb somatosensory cortex. J Comp Neurol 288:583-592.

Widmer HR, Hefti F (1994) Stimulation of GABAergic neuron differentiation by NT-4/5 in cultures of rat cerebral cortex. Dev Brain Res 80:279-284. 